\title{
ROBUST STABILITY AND DESIGN OF STATE FEEDBACK CONTROLLER FOR STRAIGHTFORWARD ACTIVE QUEUE MANAGEMENT
}

\section{MEHRDAD NOZOHOUR YAZDI AND ALI DELAVARKHALAFI*}

\author{
Department of Mathematics, Yazd University, Yazd, Iran \\ ${ }^{*}$ Corresponding author: delavarkh@yazd.ac.ir
}

\begin{abstract}
The straightforward active queue management $(A Q M)$, which is based on the prediction of arrival rate is investigated by means of state-space approach. We formulate the feedback control design problem for linearized system of additive increase multiplicative decrease $(A I M D)$ dynamic models as state-space model. Then the Lyapunov-Krasovskii method is provided to achieve the robust stability and sufficient stabilization condition and afterwards the term of linear inequality matrix $(L M I)$ is used to show the results. We present the simulation results and show the superiority of our proposed method to other control mechanisms.
\end{abstract}

\section{INTRODUCTION}

Nowadays, computer networks develop rapidly and the growth of the amount of data communicated in computer networks become an important issue in this field. Since the available capacity of the resource is usually less than the demand, congestion happens which is the main concerns to deal with in computer networks. Two effective strategies to control congestion are Transmission Control Protocol (TCP) and Active Queue Management $(A Q M)$ which they control the congestion at the end and at the hosts, respectively. TCP recognize the congestion by receiving acknowledgements and moderate the $T C P$ window sizes of senders(ref in congestion control paper). Among the categories in control systems, networked control systems (NCS) are a category in which sensors, actuators, and controllers are connected though a network. Different forms

Received 2017-10-29; accepted 2018-01-10; published 2018-09-05.

2010 Mathematics Subject Classification. 93B05, 93E15.

Key words and phrases. network congestion control; active queue management (aqm); packet arrival rate; prediction; asymptotical stability.

(C) 2018 Authors retain the copyrights of their papers, and all open access articles are distributed under the terms of the Creative Commons Attribution License. 
and investigations in NCS have been exposed in [11]. For designing the control systems, parameter effects such as delay [14] and [10], quality of service $(Q o S)$, and co-design of plant controller [7], [18], [26] and [30] are considered. The limitation on the amount of delay is assumed and there is a lot of attention on packet lost [16]. Dai et al. [8] improved the quality of service $\left(Q_{o S}\right)$ and propagate the control performance of the system.

In $T C P / A Q M$, Dynamical models for average $T C P$ window size and the queue size by including the $T C P$ networks and $A Q M$ strategies, are provided [12], [13] and [20]. In TCP/AQM models after detecting the congestion, they convey the information to end host, in which the routers drop or mark, when explicit congestion notification $(E C N)$ is activated, packets and they do all these things before the buffer overflows. Also, end hosts after redacting their congestion window size, reconcile the sending rates. Since packet-dropping causes flow-synchronization and performance depreciation, $R E D$ scheme was represented to allow to routers to facilitate TCP's management of network performance [9]. Hollot et al. [13] derived proportional and proportional-integral control for $A Q M$ schemes in which, the dynamical model developed by Misra et al. [20]. The $A Q M$ algorithms based on control theory is complicated to implement and some times parameter setting effects the system design [1] and [23]. To overcome with this, $A Q M$ algorithm with simple implementation based on prediction of arrival rate was presented in [27] and this prediction was derived from the analysis of the network congestion control.

Since these dynamical models are nonlinear, not only they are linearized at the operating point, but also this linearization is depend on a time delay which play an important role in models and their stability. Time delay can be an uncertainties for the system and robust controller such as $H_{\infty}$ could be designed for them [21]. In order to rectify the delays and the impact on system performance, the state-space models developed to formulate the control design problem [4]. Wang et al. [24] use the state-space model to derive model predictive controller and use the motion equation to linearized the system. The queue length in data buffer was predicted there and then packets at router were dropped.

When the linear system obtained, stability analysis and stabilization of linear time delay systems must be considered. The stability of linear system may be required to consider the poles of closed-loop transfer function. The routh-Hurwitz stability of a linear system, provides the stability by finding the roots of characteristic equation of the system [5], [6] and [15]. The Nyquist criterion is a graphical method and deals with the loop gain transfer function, i.e., the open loop transfer function. Hollot et al. [13] find the stability of PI and PID control and also represented that RED's queue-averaging is not beneficial by Nyquist stability. Nyquist plot specifies the factor in which the system magnitude and makes the system marginally stable [15]. That factor is called gain margin. Phase margin is obtained by phase shift which determined by Nyquist plot or bode plot [12]. Stability theory influences the system theory and engineering and also the stability implies the behavior of a system over a long time period. The asymptotic behavior of a state of the system near the 
steady-state solution like equilibrium points or operating points, helps to derive the stability of systems. The stability of equilibrium points, characterized in the sense of Lyapunov. Stability and asymptotically stability of system, were defined in [15]. The contribution of this investigation is to model state-space of the active queue management in which the drop probability policy is outspoken. The reason to go through to this designing is finding the asymptotical stability of the system which is the significant basis of the affectivity of the system. Lyapunov method is used to locate the stability conditions of an equilibrium point without solving the state equation. The remain of this paper is organized into description of the straightforward active queue management in section 2. The control model of this system and stability condition for proposed method are derived in section 3. In section 4, the illustrative examples are provided to represented the effectiveness of the method. Conclusion and suggestion of future work are located in section 5 .

\section{Prediction of the Change of packet ARrival Rate}

In order to formulate the system we first provide some relations. The system is a single congested router with a capacity $C$. Let $N, T C P$ flows labeled $i=1, \ldots, N, W_{i}(t), V_{i}(t)$ and $R_{i}(t)$ represent the congestion window size, packet sending rate and Round Trip Time $(R T T)$ of flow $T C P_{i}(i=1, \ldots, N)$ at time $t>0$, respectively. The packet arrival rate at time $t>0$ is $\lambda(t)$, then

$$
\begin{gathered}
V_{i}(t)=\frac{W_{i}(t)}{R_{i}(t)} \\
\lambda(t)=\sum_{i=1}^{n} V_{i}(t), R_{i}(t)=r_{i}+\frac{q(t)}{C},
\end{gathered}
$$

where $r_{i}$ is the Propagation of $R R T$ that we called $R T P T$ of $T C P_{i}, q(t)$ is the queue length, and the queueing delay of models is $\frac{q(t)}{C}$.

Additive Increase and Multiplicative Decrease $(A I M D)$ is the famous strategy is used here and the full description of this strategy can be found in [19]. The expectation of the increment of the arrival rate is as follows:

$$
\Delta \lambda(t)=\frac{1}{W_{i}(t) R_{i}(t)}(1-p(t-\tau))-\frac{V_{i}(t)}{2} p(t-\tau) .
$$

The moment that the packets are dropped or acknowledged to the moment that host receives the information. Here in this system $W_{i}(t), V_{i}(t)$ and $R_{i}(t)$ are unknown variables and also we assume that they have same fixed propagation delay $R_{i}(t)$ and we approximated to their expectations. The other assumptions here, are the desired value of queue occupation which is shown as $q_{\text {ref }}$ and the average value of $R T P T$ of all $T C P$ sessions which is $r$. Hence the RRT could be derived as:

$$
R_{i}(t) \approx r+\frac{q_{r e f}}{C}=R
$$


where $R$ is a $R R T$ approximation.

Afterward the expectation of the sending rate $V_{i}(t)$ and window size $W_{i}(t)$ will be expressed as follows whilst $\frac{V_{i}(t)}{\lambda(t)}$ is proportion of the packets that are generated by $T C P_{i}$,

$$
\begin{gathered}
\bar{V}(t)=\sum_{i=1}^{n} V_{i}(t) \frac{V_{i}(t)}{\lambda(t)} \geq \frac{\left(\sum_{i=1}^{n} V_{i}(t)\right)^{2}}{N \lambda(t)}=\frac{\lambda(t)}{N}, \\
\bar{W}(t)=\sum_{i=1}^{n} W_{i}(t) \frac{V_{i}(t)}{\lambda(t)} \geq \frac{\left(\sum_{i=1}^{n} V_{i}(t)\right)^{2} R}{N \lambda(t)}=\frac{\lambda(t) R}{N} .
\end{gathered}
$$

Since the system is based on packet dropping probability, so we assume it updated at every time interval and represented as $p(t)$. Also the number of arriving packets at the router is $m(t)$. Now by combing the equations (2.4-2.6), the expectation of arrival at the router is obtained below, is the objective of analysis which is provided by ( [19] and [27]) and they shown that there is a mismatch between the operating value of the queue length and desired one by their method, actually it's smaller than the desired one.

$$
\begin{aligned}
\overline{\Delta \lambda}(t+R R T) & =\sum^{m(t)} \frac{N(1-p(t))}{\lambda(t+R R T) R_{i}^{2}(t+R R T)}-\frac{\lambda(t+R R T) p(t) m(t)}{2 N} \\
& \approx \frac{N(1-p(t))}{\lambda(t+R R T) R_{i}^{2}}-\frac{\lambda(t+R R T) p(t) m(t)}{2 N}
\end{aligned}
$$

$\mathrm{Xu}$ and Sun [19] proposed the statistical method which is related to this dropping probability and they named it straightforward active queue management $(S F A Q M)$. The arrival rate when the congestion information arrive at the hosts is $\lambda(t+R R T)$ and the desired arrival rate is $\lambda_{r e f}(t+R R T)$. So in order to reach to the desired queue length $q_{r e f}$ we obtain:

$$
\begin{gathered}
\lambda(t+R R T)+\Delta \lambda(t+R R T)=\lambda_{r e f}(t+R R T), \\
\lambda_{r e f}(t+R R T)=C+\frac{\left(q_{r e f}-q(t)\right)}{\alpha} .
\end{gathered}
$$

Here, $\alpha$ is a parameter to control queue length to desired value. Hence,

$$
p(t)=\frac{\lambda(t+R R T)+\frac{m(t) N}{\lambda(t+R R T) R^{2}}-\lambda_{r e f}(t+R R T)}{\left(\frac{\lambda(t+R R T)}{2 N}+\frac{N}{\lambda(t+R R T) R^{2}}\right) m(t)}
$$

By counting the number of arriving packets $m(t)$ to estimate the arrival rate $\lambda(t)$ will be expressed as:

$$
\lambda(t)=\frac{m(t)}{\delta}
$$




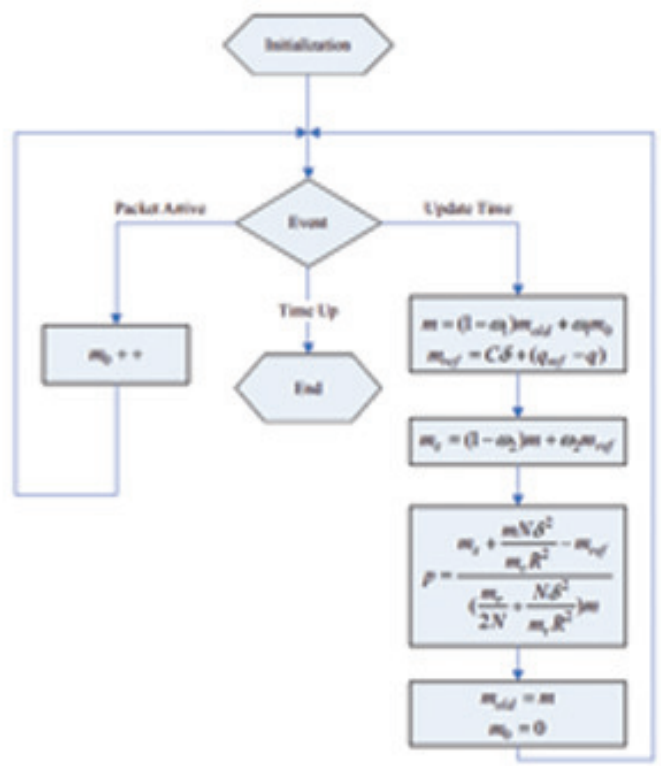

FIGURE 1. The Flowchart of $S F A Q M$ [27].

where $\delta$ is the length of the sampling period. Substituting (2.11) in (2.10) we can conclude:

$$
p(t)=\frac{m(t+R R T)+\frac{m(t) N \delta^{2}}{m(t+R R T) R^{2}}-m_{r e f}(t+R R T)}{\left(\frac{m(t+R R T)}{2 N}+\frac{N \delta^{2}}{m(t+R R T) R^{2}}\right) m(t)},
$$

where $m_{r e f}(t+R R T)$ shows as follows:

$$
m_{r e f}(t+R R T)=C \delta+\frac{\left(q_{r e f}-q(t)\right) \delta}{\alpha} .
$$

The exponential weighted moving average $(E W M A)$ was used to predict $m(t)$ by noticing that $m(t+R R T)$ is predicted as a value between $m(t)$ and $m_{r e f}(t+R R T)$, we have:

$$
\begin{aligned}
& m(t)=\left(1-\omega_{1}\right) m(t-\delta)+\omega_{1} m_{0}, \\
& m(t+R R T)=\left(1-\omega_{2}\right) m(t)+\omega_{2} m_{r e f}(t+R R T),
\end{aligned}
$$

where

- $\omega_{1}, \omega_{2}$ : weight parameter,

- $m_{0}$ : number of arriving packets during the previous interval,

- $m(t-\delta)$ : value of $m(t)$ of last sample.

The algorithm of $(S F A Q M)$ will be depicted as in Figure 1. 
Misra et al. [12], [20] and also Low et al. [19] provided the fluid-flow models to demonstrate the TCP and queue dynamics. Xu and Sun [19] claimed that their model has the same value dropping probability at the operating point. The following model as we mentioned before this is a network with one bottleneck link, with only one $T C P$ flow from each end host.

$$
\left\{\begin{array}{l}
\dot{W}_{i}(t)=\frac{W_{i}(t-R)}{R}(1-p(t-R)) \frac{1}{W_{i}(t)}-\frac{W_{i}(t-R)}{R} \frac{W_{i}(t)}{2} p(t-R), \\
\dot{q}(t)=-C+\sum_{i=1}^{N} \frac{W_{i}(t)}{R}
\end{array}\right.
$$

where $R, W_{i}(t), p(t)$ are like pervious section. Using (2.1) in this model, (2.16) changed to:

$$
\left\{\begin{array}{l}
\dot{V}(t)=\frac{V(t-R)}{R^{2} V(t)}(1-p(t-R))-\frac{1}{2} V(t-R) V(t) p(t-R), \\
\dot{q}(t)=-C+N V(t) .
\end{array}\right.
$$

The operating point will be derived by $\dot{V}(t)=0$ and $\dot{q}(t)=0$ and considering that $(V, q)$ as a state and $p$ as the input, therefore,

$$
\begin{aligned}
& \dot{V}(t)=0 \Longrightarrow p_{0}=\frac{1}{\frac{V_{0}^{2} R^{2}}{2}+1}, \\
& \dot{q}(t)=0 \Longrightarrow V_{0}=\frac{C}{N}
\end{aligned}
$$

Now equation (2.12) is concluded as follows by substituting equation (2.11), (2.13) and (2.14):

$$
p(t)=\frac{\left[\left(1-\omega_{2}\right) \lambda(t)+\omega_{2} \lambda_{r e f}(t+\tau)\right]^{2}+\frac{N \delta \lambda(t)}{R^{2}}-\lambda(t) \lambda_{r e f}(t+\tau)}{\left(\frac{\left[\left(1-\omega_{2}\right) \lambda(t)+\omega_{2} \lambda_{r e f}(t+\tau)\right]^{2}}{2 N}+\frac{N}{R^{2}}\right) \lambda(t) \delta} .
$$

Then the packet dropping probability will be as follows when the system is at the steady state, and the queue occupation, arrival rate and desired arrival rate are stabilized at $q_{r e f}$ and $C$ (link capacity), respectively:

$$
p_{0}=\frac{1}{\frac{C^{2} R^{2}}{2 N^{2}}+1} .
$$

As we mentioned this value is like the same value as Low et al. [19] calculated in their model.

For linearizing the above equation the right hand side of equation (2.17) can be written as follows:

$$
\left\{\begin{array}{l}
f\left(V, V_{\tau}, p_{\tau}\right)=\frac{V_{\tau}}{R^{2} V}\left(1-p_{\tau}\right)-\frac{1}{2} V_{\tau} V p_{\tau} \\
g(V)=-C+N V \\
h(\dot{q}, q)=\frac{\left[\left(1-\omega_{2}\right) \lambda+\omega_{2} \lambda_{r e f}\right]^{2}+\frac{N \delta \lambda}{R^{2}}-\lambda \lambda_{r e f}}{\left(\frac{\left[\left(1-\omega_{2}\right) \lambda+\omega_{2} \lambda_{r e f}\right]^{2}}{2 N}+N / R^{2}\right) \lambda \delta}
\end{array}\right.
$$


where $V_{\tau}(t)=V(t-\tau)$ and $\lambda, \lambda_{\text {ref }}$ can be shown as:

$$
\left\{\begin{array}{l}
\lambda(t)=\sum_{i=1}^{N} V_{i}(t)=\dot{q}(t)+C \\
\lambda_{r e f}=C+\frac{\left(q_{r e f}-q(t)\right)}{\alpha}
\end{array}\right.
$$

Deriving partial derivatives at the operating point in (2.23) we have:

$$
\begin{aligned}
& \frac{\partial f}{\partial V}=-\frac{V_{0}\left(1-p_{0}\right)}{R^{2} V_{0}^{2}}-\frac{1}{2} V_{0} p_{0}=-\frac{1-p_{0}}{R^{2} V_{0}}-\frac{1}{2} V_{0} p_{0}= \\
& -\frac{N}{R^{2} C} \frac{\frac{C^{2} R^{2}}{2 N^{2}}}{\frac{C^{2} R^{2}}{2 N^{2}}+1}-\frac{1}{2} \frac{C}{N} \frac{1}{\frac{C^{2} R^{2}}{2 N^{2}}+1}=-\frac{C}{N} \frac{1}{\frac{C^{2} R^{2}}{2 N^{2}}+1}, \\
& \frac{\partial f}{\partial V_{\tau}}=\frac{\left(1-p_{0}\right)}{R^{2} V_{0}}-\frac{1}{2} V_{0} p_{0}=\frac{N}{R^{2} C} \frac{\frac{C^{2} R^{2}}{2 N^{2}}}{\frac{C^{2} R^{2}}{2 N^{2}}+1}-\frac{1}{2} \frac{C}{N} \frac{1}{\frac{C^{2} R^{2}}{2 N^{2}}+1}=0 \\
& \frac{\partial f}{\partial p_{\tau}}=-\frac{1}{2} V^{2}=-\frac{C^{2}}{2 N^{2}} \\
& \frac{\partial g}{\partial V}=N \\
& \frac{\partial h}{\partial \dot{q}}=\frac{\partial h}{\partial \lambda} \frac{\partial \lambda}{\partial \dot{q}}=\frac{\partial h}{\partial \lambda} \\
& \frac{\partial h}{\partial q}=\frac{\partial h}{\partial \lambda_{r e f}} \frac{\partial \lambda_{r e f}}{\partial q}=-\frac{1}{\alpha} \frac{\partial h}{\partial \lambda_{r e f}} .
\end{aligned}
$$

In order to obtain the two last equations, let $a$ and $b$ be the numerator and denominator of $h$, respectively. So,

$$
\left\{\begin{array}{l}
\frac{\partial a}{\partial \lambda}=2\left(1-\omega_{2}\right) C+\frac{N \delta}{R^{2}}-C \\
\frac{\partial b}{\partial \lambda}=\left(3 C^{2}-2 \omega_{2}^{2} C^{2}\right) \frac{\delta}{2 N}+\frac{N \delta}{R^{2}} \\
\frac{\partial a}{\partial \lambda_{r e f}}=2 \omega_{2} C-C \\
\frac{\partial b}{\partial \lambda_{r e f}}=\frac{\omega_{2} C^{2}}{N}
\end{array}\right.
$$

Now we have:

$$
\left\{\begin{array}{l}
\frac{\partial h}{\partial \lambda}=\frac{\frac{\partial a}{\partial \lambda} b-\frac{\partial b}{\partial \lambda} a}{b^{2}} \\
\frac{\partial h}{\partial \lambda_{r e f}}=\frac{\frac{\partial a}{\partial \lambda_{r e f}} b-\frac{\partial b}{\partial \lambda_{r e f}} a}{b^{2}}
\end{array}\right.
$$


Thus the linearizing form is as follows:

$$
\left\{\begin{array}{l}
\delta \dot{V}(t)=-\frac{C}{N\left(\frac{C^{2} R^{2}}{2 N^{2}}+1\right)} \delta V(t)-\frac{C^{2}}{2 N^{2}} \delta p(t-R) \\
\delta \dot{q}(t)=N \delta V(t) \\
\delta p(t)=\frac{\partial p}{\partial \lambda} \delta \dot{q}(t)-\frac{1}{\alpha} \partial p \partial \lambda_{r e f} \delta q(t)
\end{array}\right.
$$

\section{Designing Control model of TCP/AQM}

Model (2.22) can be formulated as:

$$
\left\{\begin{array}{l}
\dot{x}_{p}=A_{p} x(t)+B_{p} u(t-R)+B_{d} u(t) \\
y_{p}=c_{p} x(t)
\end{array}\right.
$$

in which $x(t)=[\delta V(t) \delta q(t)], \delta p(t)=u(t), y(t)=\delta q(t)$ and

$$
\begin{gathered}
A_{p}=\left[\begin{array}{cc}
-\frac{C}{N\left(\frac{C^{2} R^{2}}{2 N^{2}}+1\right)} & 0 \\
N & \frac{1}{\alpha} \frac{D_{2}}{D_{1}}
\end{array}\right] \\
B_{p}=\left[\begin{array}{c}
\frac{C^{2}}{2 N^{2}} \\
0
\end{array}\right] \\
B_{d}=\left[\begin{array}{c}
0 \\
\frac{1}{D_{1}}
\end{array}\right]
\end{gathered}
$$

where $D_{1}=\frac{\partial p}{\partial \lambda}=\frac{\partial h}{\partial \lambda}$ and $D_{2}=\frac{\partial p}{\partial \lambda_{r e f}}=\frac{\partial h}{\partial \lambda_{r e f}}$ and also,

$$
\delta \dot{q}=\left(\frac{\partial p}{\partial \lambda}\right)^{-1} \delta p(t)+\frac{1}{\alpha}\left(\frac{\partial p}{\partial \lambda_{r e f}}\right)^{-1} \delta q(t)=\frac{1}{D_{1}} \delta p(t)+\frac{1}{\alpha} \frac{D_{2}}{D_{1}} \delta q .
$$

We can write the state-space as:

$$
\left\{\begin{array}{l}
\dot{x}_{p}=A_{p} x(t)+B_{p} u(t-R) \\
y_{p}=c_{p} x(t) \\
u(t)=D_{p} \dot{x}(t)+D_{c} x(t)
\end{array}\right.
$$

where $D_{p}=\left[0 u_{\lambda}\right], D_{c}=\left[0 \frac{-1}{\alpha u_{r e f}}\right]$ and

$$
\begin{gathered}
A_{p}=\left[\begin{array}{cc}
-\frac{C}{N\left(\frac{C^{2} R^{2}}{2 N^{2}}+1\right)} & 0 \\
N & 0
\end{array}\right], \\
B_{p}=\left[\begin{array}{c}
\frac{C^{2}}{2 N^{2}} \\
0
\end{array}\right] .
\end{gathered}
$$


As we can see the model (3.1) is a state-dependent delay differential equation with delay in control. Assuming this model uses a state feedback controller $u_{n}(t)=K_{n} x_{p}(t)$ i.e., $u_{n}(t)=K_{n 1} \delta V(t)+K_{n 2} \delta q(t)$, this equation will changed to:

$$
\begin{gathered}
\left\{\begin{array}{l}
\dot{x}_{p}=A_{p} x_{p}(t)+B_{p} K_{p} x_{p}(t-R)+B_{d} K_{n} x_{p}(t), \\
y_{p}=c_{p} x_{p}(t),
\end{array}\right. \\
\left\{\begin{array}{c}
\dot{x}_{p}=\left(A_{p}+B_{d} K_{n}\right) x_{p}(t)+B_{p} K_{p} x_{p}(t-R), \\
y_{p}=c_{p} x_{p}(t),
\end{array}\right. \\
A=\left[\begin{array}{c}
-\frac{C}{N\left(\frac{C^{2} R^{2}}{2 N^{2}}+1\right)} \\
N+\frac{1}{D_{1}} \\
\frac{1}{\alpha} \frac{D_{2}}{D_{1}}
\end{array}\right] \\
A_{d}=\left[\begin{array}{c}
-\frac{C^{2}}{2 N^{2}} \\
0
\end{array}\right]
\end{gathered}
$$

For proofing the stability of state-dependent system which is describe by (3.4), the following theorems are applied whilst stability conditions will be constructed to insure the stability and give the variables of pervious system. First the Lemma which is proposed by Lin [17] is provided as follows and will be used in the proof of aforementioned theorems.

Lemma 3.1. If there is exist arbitrary matrices $X_{11}, X_{12}, X_{13}, X_{22}, X_{23}$ and $X_{33}$ such that

$$
X=\left[\begin{array}{ccc}
X_{11} & X_{12} & X_{13} \\
X_{12}^{T} & X_{22} & X_{23} \\
X_{13}^{T} & X_{23}^{T} & X_{33}
\end{array}\right] \geq 0
$$

then we obtain

$$
\begin{aligned}
& -\int_{t-R R T(t)}^{t} \dot{x}^{T}(s) X_{33} \dot{x}(s) d s \leq \\
& \int_{t-R R T(t)}^{t}\left[x^{T}(t) x^{T}(t-R R T(T)) \dot{x}^{T}(s)\right]\left[\begin{array}{ccc}
X_{11} & X_{12} & X_{13} \\
X_{12}^{T} & X_{22} & X_{23} \\
X_{13}^{T} & X_{23}^{T} & 0
\end{array}\right]\left[\begin{array}{c}
x(t) \\
x(t-R R T(t)) \\
\end{array}\right] d s .
\end{aligned}
$$

Theorem 3.1. Let the scalars $h>0$ and $\mu<1$, the state-dependent delay system (3.3) is asymptotically stable, if the condition $(\nabla R R T . \dot{x}(t)=\lambda(t)<\mu<1)$ is satisfied for every $t$ and there exist positive-definite 
symmetric matrices $P, Q$ and $R$, and a semi positive-definite matrix

$$
X=\left[\begin{array}{ccc}
X_{11} & X_{12} & X_{13} \\
X_{21} & X_{22} & X_{23} \\
X_{31} & X_{32} & X_{33}
\end{array}\right],
$$

such that the following Linear matrix Inequality(LMI) holds

$$
A_{p}=\left[\begin{array}{ccc}
E_{11} & E_{12} & h A^{T} R \\
* & E_{22} & h A_{d}^{T} R \\
* & * & -h R
\end{array}\right]<0,
$$

$R-X_{33} \geq 0$,

where

$$
\begin{aligned}
& E_{11}=A^{T} P+P A+Q+(1-\mu)\left(X_{13}+X_{13}^{T}+h X_{11}\right), \\
& E_{12}=P A_{d}+(1-\mu)\left(-X_{13}+X_{23}^{T}+h X_{12}\right), \\
& E_{22}=(1-\mu)\left(-Q-X_{23}-X_{23}^{T}+h X_{22}\right) .
\end{aligned}
$$

Lyapunov stability theory are concept that applied to investigate the ability of these theorems. Shevitz and Paden were proceeded the lyapunov stability for nonsmooth systems [22] and also, Zhang et al. [28] utilized feedback control system and provided the stability for networked control systems where their approach is used to establish the stability. Also, Lyapunov-Krasovskii functional and schur complement are using to proof of theorem.

Proof: The Lyapunov-Krasovskii that we use here, is as follows:

$$
V\left(x_{t}\right)=x^{T}(t) P x(t)+\int_{t-R R T}^{t} x^{T}(s) Q x(s) d s+\int_{-R R T}^{0} \int_{t+\theta}^{t} \dot{x}^{T}(s) R \dot{x}(s) d s d \theta .
$$

Obviously, this Lyapunov functional candidate is positive definite. Now we calculate the derivative of this functional:

$$
\begin{aligned}
\dot{V}\left(x_{t}\right) & =\dot{x}^{T}(t) P x(t)+x^{T} P \dot{x}(t)+x^{T}(t) Q x(t)-(1-R \dot{R} T(x)) Q x(t-R R T(x)) \\
& +R R T(x) \dot{x}^{T}(t) R \dot{x}(t)-(1-R \dot{R} T(x)) \int_{t-R R T(x)}^{t} \dot{x}^{T}(s) R \dot{x}(s) d s .
\end{aligned}
$$


By substituting (3.7) to (3.8) yields

$$
\begin{aligned}
\dot{V}\left(x_{t}\right) & =x^{T}(t)\left[A^{T} P+P A+Q\right] x(t)+x^{T}(t) P A_{d} x(t-R R T(x)) \\
& +(1-\lambda(t)) x^{T}(t-R R T(x)) Q x(t-R R T(x))+R R T(x) \dot{x}^{T}(t) R \dot{x}(t) \\
& -(1-R \dot{R} T(x)) \int_{t-R R T(x)}^{t} \dot{x}^{T}(s)\left(R-X_{33}\right) \dot{x}(s) d s \\
& -(1-R \dot{R} T(x)) \int_{t-R R T(x)}^{t} \dot{x}^{T}(s) X_{33} \dot{x}(s) d s .
\end{aligned}
$$

The Leibniz-Newton formula used here and the assumption on $R \dot{R} T(x)<\mu$ and $R R T(x)<h$. So we have

$$
\begin{aligned}
& -(1-\mu) \int_{t-R R T(x)}^{t} \dot{x}^{T}(s) X_{33} \dot{x}(s) d s \leq(1-\mu) \int_{t-R R T(x)}^{t}\left[x^{T} x^{T}(t-R R T(x)) \dot{x}(s)\right] \\
& +\left[\begin{array}{ccc}
X_{11} & X_{12} & X_{13} \\
X_{21} & X_{22} & X_{23} \\
X_{31} & X_{32} & X_{33}
\end{array}\right]\left[\begin{array}{c}
x^{T} \\
x^{T}(t-R R T(x)) \\
\dot{x}(s)
\end{array}\right] \leq(1-\mu) h x^{T}(t) X_{11} x(t)+x^{T} h X_{12} x(t-R R T(x))+ \\
& +x^{T} X_{13} \int_{t-R R T(x)}^{t} \dot{x}(s) d s+h x^{T}(t-R R T(x)) X_{12}^{T} x(t)+h x^{T}(t-R R T(x)) X_{22} x(t-R R T(x)) \\
& +\quad X_{23}^{T} x(t-R R T(x)) \int_{t-R R T(x)}^{t} \dot{x}(s) d s=(1-\mu)\left[x^{T}(t) h X_{11} x(t)+x^{T}(t) h X_{12} x(t-R R T(x))\right. \\
& +\quad x^{T}(t) X_{13} x(t)-x(t-R R T(x)) X_{13}^{T} x(t)+x^{T}(t-R R T(x)) h X_{12}^{T} x(t) \\
& +\quad x^{T}(t-R R T(x)) h X_{22} x(t-R R T(x))+x^{T}(t-R R T(x)) X_{23} x(t)-x^{T}(t-R R T(x)) X_{23} x(t-R R T(x)) \\
& \left.+\quad x^{T}(t) X_{13}^{T} x(t)-x^{T}(t) X_{13}^{T} x(t-R R T(x))+x(t) X_{23}^{T} x(t-R R T(x))-x(t-R R T(x)) X_{23}^{T} x(t-R R T(x))\right] .
\end{aligned}
$$

By substituting in (3.9), following equation will be concluded:

$$
\dot{V}\left(x_{t}\right)<\xi^{T}(t) Z \xi(t)-(1-\mu) \int_{t-R R T(x)}^{t} \dot{x}^{T}(s)\left(R-X_{33}\right) \dot{x}(s) d s
$$

where $\xi^{T}(t)=\left[x^{T} x^{T}(t-R R T(x))\right]$ and

$$
\left[\begin{array}{cc}
z_{11} & z_{12} \\
0 & z_{22}
\end{array}\right]
$$




$$
\begin{aligned}
& z_{11}=A^{T} P+P A+Q+(1-\mu)\left(X_{13}+X_{13}^{T}+h X_{11}\right)+h A^{T} R A, \\
& z_{12}=P A_{d}+(1-\mu)\left(-X_{13}+X_{23}^{T}+h X_{12}\right)+h A^{T} R A_{d}, \\
& z_{22}=(1-\mu)\left(-Q-X_{23}-X_{23}^{T}+h X_{22}\right)+h A_{d}^{T} R A_{d} .
\end{aligned}
$$

In order to achieve to negative derivative of Lyapunov functional, we use the schur complement, (i.e. $\left.Z / z_{11}=z_{22}-z_{12} z_{11}^{-1}<0\right)$ and LMI conditionals. So the asymptotically stability will be derived.

Using the proof of this theorem and substituting AQM model on stability condition yields:

$$
\begin{aligned}
\dot{V}(x) & =x^{T}(t)\left[\left[\begin{array}{cc}
-\frac{C}{N\left(\frac{C^{2} R^{2}}{2 N^{2}}+1\right)} & 0 \\
\left(N+\frac{1}{D_{1}}\right) K_{n} & \frac{1}{\alpha} \frac{D_{2}}{D_{1}}
\end{array}\right]^{T} P+P\left[\begin{array}{cc}
-\frac{C}{N\left(\frac{C^{2} R^{2}}{2 N^{2}}+1\right)} & 0 \\
\left(N+\frac{1}{D_{1}}\right) K_{n} & \frac{1}{\alpha} \frac{D_{2}}{D_{1}}
\end{array}\right]+Q\right] x(t) \\
+ & x^{T} P\left[\begin{array}{c}
\frac{C^{2}}{2 N^{2}} K_{p} \\
0
\end{array}\right] x(t-R R T(x))+x^{T}(t-R R T(x))\left[\frac{C^{2}}{2 N^{2}} K_{p} 0\right] Q x(t-R R T(x)) \times \\
& R R T(x) \dot{x}^{T}(t) R \dot{x}(t)-(1-R \dot{R} T(x)) \int_{t-R R T(x)}^{t} \dot{x}^{T}(s)\left(R-X_{33}\right) \dot{x}(s) d s \\
- & (1-R \dot{R} T(x)) \int_{t-R R T(x)}^{t} \dot{x}^{T}(s) X_{33} x(s) d s .
\end{aligned}
$$

So the stability of Lyapunov functional, we obtain:

$$
\begin{gathered}
\dot{V}(x)<\left[x^{T}(t) x^{T}(t-R R T(x))\right] \times\left[\begin{array}{cc}
M & N \\
0 & F
\end{array}\right] \times\left[x^{T} x^{T}(t-R R T(x))\right]^{T} \\
-(1-\mu) \int_{t-R R T(x)}^{t} \dot{x}^{T}(s)\left(R-X_{33}\right) \dot{x}(s) d s<0, \\
{\left[\begin{array}{cc}
-\frac{C}{N\left(\frac{C^{2} R^{2}}{2 N^{2}}+1\right)} & 0 \\
\left(N+\frac{1}{D_{1}}\right) K_{n} & \frac{1}{\alpha} \frac{D_{2}}{D_{1}}
\end{array}\right]^{T} P+P\left[\begin{array}{cc}
-\frac{C}{N\left(\frac{C^{2} R^{2}}{2 N^{2}}+1\right)} & 0 \\
\left(N+\frac{1}{D_{1}}\right) K_{n} & \frac{1}{\alpha} \frac{D_{2}}{D_{1}}
\end{array}\right]+(1-\mu)\left(X_{13}+X_{13}^{T}+h X_{11}\right)} \\
+h\left[\begin{array}{ccc}
-\frac{C}{N\left(\frac{C^{2} R^{2}}{2 N^{2}}+1\right)} & 0 \\
\left(N+\frac{1}{D_{1}}\right) K_{n} & \frac{1}{\alpha} \frac{D_{2}}{D_{1}}
\end{array}\right]^{T} R\left[\begin{array}{cc}
-\frac{C}{N\left(\frac{C^{2} R^{2}}{2 N^{2}}+1\right)} & 0 \\
\left(N+\frac{1}{D_{1}}\right) K_{n} & \frac{1}{\alpha} \frac{D_{2}}{D_{1}}
\end{array}\right]<0,
\end{gathered}
$$

where,

$$
\begin{aligned}
M & =A^{T} P+P A+(1-\mu)\left(X_{13}+X_{13}^{T}+h X_{11}\right)+h A^{T} R A \\
N & =P A_{d}+(1-\mu)\left(X_{13}+X_{23}^{T}+h X_{12}\right)+h A R A_{d}, \\
F & =(1-\mu)\left(X_{23}+X_{23}^{T}+h X_{22}\right)+h A_{d}^{T} R A_{d} .
\end{aligned}
$$


Simple calculation leads the following equation:

$$
\left(N+\frac{1}{D_{1}}\right) K_{n} P_{21}+2\left(\frac{1}{\alpha} \frac{D_{2}}{D_{1}} P_{22}\right)+h\left(\frac{1}{\alpha} \frac{D_{2}}{D_{1}}\right)^{2} R_{22}<2\left(\frac{C}{N\left(\frac{C^{2} R^{2}}{2 N}+1\right)}\right) P_{11}-h\left(\frac{C}{N\left(\frac{C^{2} R^{2}}{2 N}+1\right)}\right)^{2} R_{11}
$$

Theorem 3.2. For any given $h$ and $\mu<1$, the state dependent delay is asymptotically stabilized via the state feedback controller $u(t)=\tilde{K} x(t)$, if the condition $(\nabla R R T . \dot{x}(t)=\lambda(t)<\mu<1)$ satisfies for every $t$ and there exists positive definite symmetric matrices $W, U, G$ and a matrix $Y$ with appropriate dimensions and a semi-positive definite matrix,

$$
T=\left[\begin{array}{ccc}
T_{11} & T_{12} & T_{13} \\
T_{21} & T_{22} & T_{23} \\
T_{31} & T_{32} & T_{33}
\end{array}\right] \geq 0
$$

such that the following LMI holds,

$$
\Gamma=\left[\begin{array}{ccc}
\Gamma_{11} & \Gamma_{12} & h W\left[\begin{array}{cc}
-\frac{C}{N\left(C^{2} R^{2} / 2 N^{2}+1\right)} & 0 \\
\left(N+\frac{1}{D_{1}}\right) K_{n} & \frac{1}{\alpha} \frac{D_{2}}{D_{1}}
\end{array}\right] \\
* & \Gamma_{22} & {\left[\begin{array}{c}
\frac{C^{2}}{2 N^{2}} K_{p} \\
0
\end{array}\right]+h Y B_{d}^{T}} \\
* & * & -h G
\end{array}\right]<0
$$

and $W-T_{33} \geq 0$, where,

$$
\begin{aligned}
\Gamma_{11} & =W\left[\begin{array}{cc}
-\frac{C}{N\left(C^{2} R^{2} / 2 N^{2}+1\right)} & 0 \\
\left(N+\frac{1}{D_{1}}\right) K_{n} & \frac{1}{\alpha} \frac{D_{2}}{D_{1}}
\end{array}\right]^{T}+\left[\begin{array}{cc}
-\frac{C}{N\left(C^{2} R^{2} / 2 N^{2}+1\right)} & 0 \\
\left(N+\frac{1}{D_{1}}\right) K_{n} & \frac{1}{\alpha} \frac{D_{2}}{D_{1}}
\end{array}\right] W+(1-\mu)\left(T_{13}+T_{13}^{T}+h T_{11}\right), \\
\Gamma_{12} & =\left[\begin{array}{c}
\frac{C^{2}}{2 N^{2}} K_{p} \\
0
\end{array}\right] W+\left[\begin{array}{c}
0 \\
\frac{1}{D_{1}}
\end{array}\right] Y+(1-\mu)\left(-T_{13}+T_{23}^{T}+h T_{12}\right), \\
\Gamma_{22} & =(1-\mu)\left(-U-T_{23}-T_{23}^{T}+h T_{22}\right),
\end{aligned}
$$

and a stabilizing gain will be driven by:

$$
\left[\begin{array}{cc}
K_{p} & 0 \\
0 & K_{n}
\end{array}\right]=Y W^{-1} .
$$


Proof: Since matrix $A_{d}$ including controller gains, and its needs to be disassembled, so it should be calculate and we will be factorized matrix $A_{d}$ as,

$$
\left[\begin{array}{cc}
\frac{C^{2}}{2 N^{2}} & \\
0 & K_{p}
\end{array}\right] .
$$

Replacing in $L M I$ condition and multiplying to the both sides of that by diag $P^{-1}, R^{-1}$, defining $W=P^{-1}$, $G=R^{-1}, P^{-1} Q P^{-1}=U, \tilde{K} W=Y, P^{-1} X_{i j} P^{-1}=T_{i j}$ and $\left[R^{-1} P^{-1}\right]\left[R-X_{33}\right]^{T}, P^{-1}=W-T_{33}$ lead to the equation in theorem and the state feedback controller gain then can be found from $\tilde{K}=Y W^{-1}$ The rate at which the queue length grows when the buffer is nonempty in which Athuraliya et al. [2] mentioned as mismatch. Also, the $A Q M$ method uses state feedback, but $V_{k}-V$ states might be not available at routers and it makes some difficulties in real network.

An approximation uses here to conquer this problem. The control signal for network is $u_{n}(t)=K_{n} x_{n}(t)$ which yields:

$$
\delta p(t)=K_{n} x_{n}(t)=\left[\begin{array}{ll}
K_{n 1} & K_{n 2}
\end{array}\right][\delta q \delta V]^{T}
$$

As [29], the second term of the state vector is investigated:

$$
\begin{aligned}
& x_{2}=V-V_{0}=V-\frac{\tau_{0} C}{N}=\frac{\tau_{0}}{N}\left(\frac{V N}{\tau_{0}}-C\right), \\
& \frac{\tau_{0}}{N}[\text { flowrate }-C]=\frac{\tau_{0}}{N} * \text { rate of mismatch } .
\end{aligned}
$$

This mismatch can be approximated by $\dot{q}(t)$ and it turns to:

$$
\delta p(t)=K_{n 1} \cdot\left(q-q_{0}\right)+K_{n 2} \cdot \frac{\tau_{0}}{N} \dot{q}(t)
$$

\section{Numerical Simulation}

Some Examples of control systems have been simulated here.The parameters $\omega_{1}, \omega_{2}, \alpha$ and $\delta$ will be calculated according to stability condition that we provided in pervious section. [27] determined that $q_{\text {ref }}$ as reference queue length can be set 500 packets which is the desired value. Also, they set $R=0.1889 s$ and RTPT $=0.1 s$. Following examples consider the different network condition, actually we alter the network condition. We will see in following examples that all these parameters satisfy the stability condition corresponding to these network systems.

The network topology that we use here is dumb-bell which the link between router $B$ and router $C$ is the 


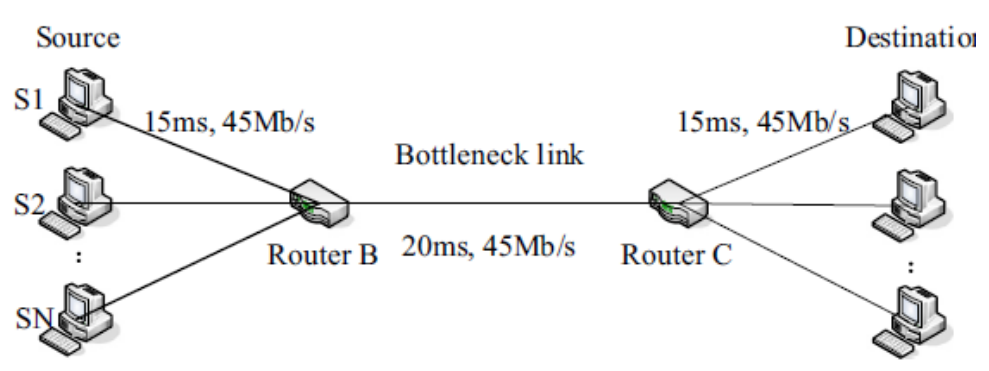

Figure 2. The Network Topology [27].

bottleneck link and it's depicted in figure (2). TCP/Reno is the policy that the sources use. All routers use Droptail except router $B$ where it exploit $S F A Q M$ to control queue length. We use the network control system with following features:

- Propagation delay is set to $200 \mathrm{~ms}$,

- Nominal load is 1000 sessions,

- Desired queue length is set 100 packets,

- Buffer capacity is set 300 packets.

In following examples the parameters corresponding to $S F A Q M$ policy will be derived according to the stability condition proposed in this paper. It must mentioned that $\delta$ is set to $0.00625 \mathrm{~s}$. The results explain the effectiveness and good performance of the presented method and also show the these parameters speed of convergence of $S F A Q M$ under this stability condition.

Example 4.1. Consider the following system which is proposed by Huang and Ngnuang [14]

$$
A_{p}=\left[\begin{array}{cc}
0 & 1 \\
0 & 0.1
\end{array}\right], B_{p}=\left[\begin{array}{c}
0 \\
0.1
\end{array}\right], B_{d}=\left[\begin{array}{c}
0 \\
0.01
\end{array}\right]
$$

Applying control algorithm proposed in [3] and also considering the theorem (3.2) and relation (3.16), the controller gains are derived as:

$$
\begin{aligned}
& u_{p}(t)=\left[\begin{array}{ll}
-1.4176 & -5.6137
\end{array}\right] x_{p}(t), \\
& u_{n}(t)=\left[\begin{array}{ll}
0.0002 & 0.0072
\end{array}\right] x_{n}(t) .
\end{aligned}
$$

The state trajectories of the system which is shown in figure (3), is derived by applying the obtained control into the aforementioned network control system with initial values $x_{0}=\left[\begin{array}{ll}1 & 1\end{array}\right]^{T}$. Also the queue length 


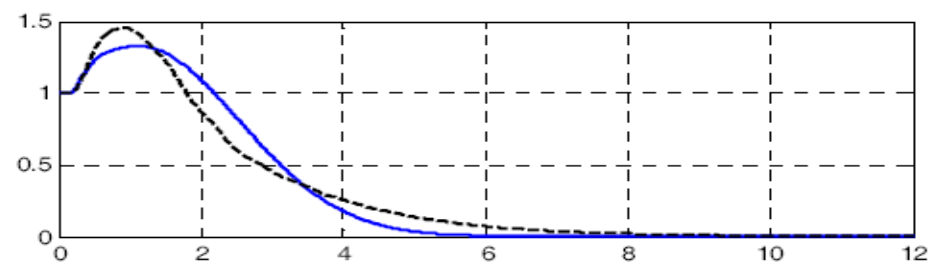

FIGURE 3. The state trajectories of the proposed system .

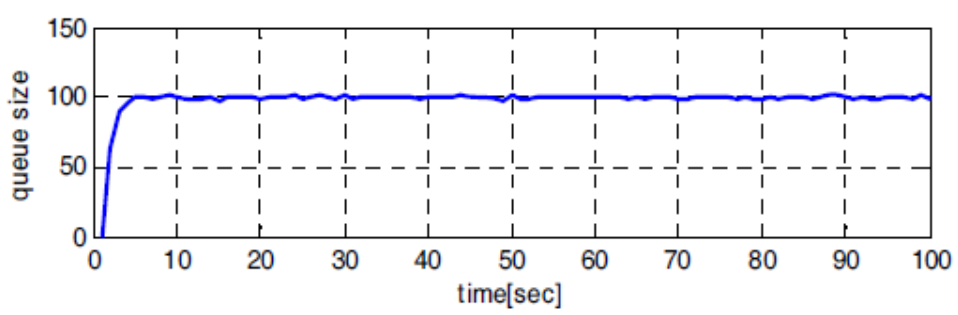

FIGURE 4. The queue length of network control system.

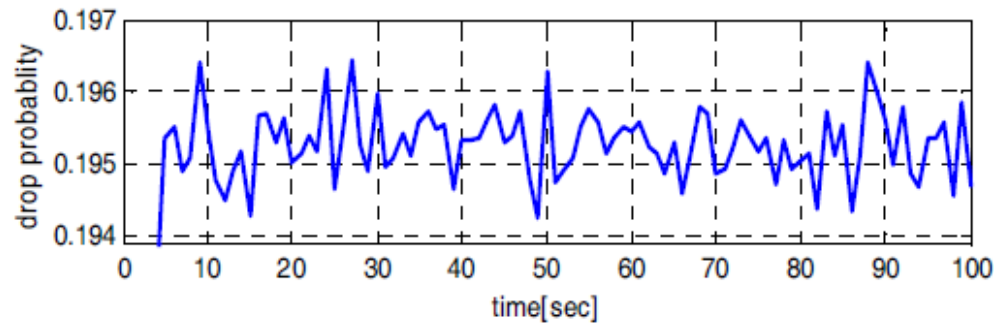

FIGURE 5. The drop probability as a control law.

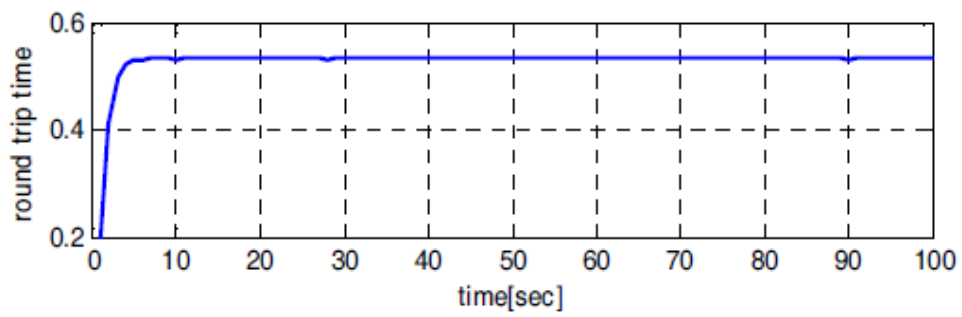

Figure 6 . The state dependent delay.

is represented in figure (4). Figure (5), shows the drop probability of system and state dependent delay is depicted in figure (6). The stability of the network control system by proposed method is noticeable by this figures. As shown, the queue length and state dependent delay keep stayed around value. The result is coincide with the result of Azadegan et al. [3], which is represent the affectivity of our proposed method. We must mentioned that we use LMI Toolbox at the MATLAB to obtain this control law. 
Example 4.2. Extending the unstable system where proposed in [25], as follows:

$$
\dot{x}(t)=\left[\begin{array}{ccc}
-1 & 0 & -0.5 \\
1 & -0.5 & 0 \\
0 & 0 & 0.5
\end{array}\right] x(t)+\left[\begin{array}{c}
0 \\
0 \\
0.1
\end{array}\right] x(t-R)+\left[\begin{array}{l}
0 \\
0 \\
1
\end{array}\right] .
$$

,

and by applying the theorem (3.2), the controlled gain is obtained:

$$
\begin{aligned}
& u_{p}(t)=\left[\begin{array}{lll}
0 & 0 & -0.8762
\end{array}\right] x_{p}(t), \\
& u_{n}(t)=\left[\begin{array}{ll}
0.0003 & 0.0018
\end{array}\right] x_{n}(t),
\end{aligned}
$$

with initial state $x_{0}=\left[\begin{array}{lll}-5 & 0 & 5\end{array}\right]^{T}$. Same as [3] and [25], the state components convergence to zero in faster time as expected. It must be noticed that the asymptotic stability of the aforementioned system is provided by [14] and it's consider the effect of transmission delay will affect the system.

\section{Conclusion}

The stabilizing feedback control based on the state-space method for the straightforward $A Q M$ system has been investigated in this paper. We provided the state-feedback control and to regulate the SFAQM dynamical system, we derived the explicit delay compensation structures. The robust stability was obtained by Lyapunov-Krasovskii method and the stabilization condition has been presented in the form of LMIs. Using NS2 software and MATLAB toolbox, simulation results have been verified the effectiveness and superiority of our method compared with other $A Q M$ schemes on queue stability. In the future work we will use optimal control theory and $M-$ Matrix form to induce more robust $A Q M$ schemes.

\section{REFERENCES}

[1] A. E. Abharian, H. Khaloozadeh, and R. Amjadifard, Genetic-sigmoid random early detection covariance control as a jitter controller, IET Control Theory Appl. 6 (2012), 327 - 334,.

[2] S. Athuraliya, S., S. Low, V. Li, and Q. Yin, REM: active queue management, IEEE Network Magazine, 15 (2001), 48-53,.

[3] M. Azadegan, M. T. Beheshti, and B. Tavassoli, Using AQM for performance improvement of networked control systems, Int. J. Control Autom. Syst. 13 (2015), 764-772.

[4] T. Azuma, T. Fujita, and M. Fujita, Congestion Control for TCP/AQM Networks using State Predictive Control, Electr. Eng. Japan, 156 (2006), 41-47.

[5] H. W. Bode, Network analysis and feedback amplifier design, Van Nostrand, New York (1945).

[6] R. V. Churchill, Brown, J. V., and Verhy, R. F., Complex variable and applications, McGraw-Hill, New York, (1976).

[7] D. B. Dacic, and D. Nesic, Quadratic stabilization of linear networked control systems via simultaneous protocol and controller design, Automatica, 43 (2007), 1145-1155. 
[8] S. L. Dai, H. Lin, and S. S. Ge, Scheduling and control codesign for a collection of networked control systems with uncertain delays, IEEE Trans.Control Syst. Technol., 18 (2010), 66-78,.

[9] S. Floyd, and V. Jacobson, Random Early Detection Gateways for Congestion Avoidance, IEEE/ACM Trans. Networking, 1 (1993), 397-413.

[10] Y. Ge, J. Wang, and C. Li, Robust stability conditions for DMC controller with uncertain time delay, Int. J. Control Autom. Syst., 12 (2) (2014), 241-250.

[11] R. A. Gupta, and M. Y. Chow, Networked control system: Overview and research trends, IEEE Trans.Ind. Electron., 57 (7) (2010), 2527-2535.

[12] C. V. Hollot, V. Misra, D. Towsley, and W. Gong On Designing Improved Controllers for AQM Routers Supporting TCP Flows, Proc. IEEE INFOCOM, 3 (2001), 1726-34.

[13] C. V. Hollot, V. Misra, D. Towsley, and W. Gong Analysis and Design of Controllers for AQM Routers Supporting TCP Flows, IEEE Trans. Autom. Control, 47 (6) (2002), 945-959.

[14] D. Huang, and S. K. Nguang, State feedback control of uncertain networked control systems with random time delays, IEEE Trans. Autom. Control, 53 (3) (2008), 829-834.

[15] W. S. Levine, The control handbook, CRC Press, IEEE Press, Boca Raton, New York , (1996).

[16] H. Li, and Y. Shi, Network-based predictive control for constrained nonlinear systems with two-channel packet dropouts, IEEE Trans. Ind. Electron., 61 (10) (2014), 1574-1582,.

[17] P. Liu, Robust exponential stability for uncertain time-varying delay systems with delay dependence, J. Franklin Inst. 346 (3) (2009), 958-968.

[18] X. Liu, and A. Goldsmith, Wireless network design for distributed control, 43rd IEEE Conf. on Decision and Control, pp. 2823-2829, (2004).

[19] S. H. Low, F. Paganini, and J. C. Doyle, Internet Congestion Control, 43rd IEEE Control Syst. pp. 28-43, (2002).

[20] V. Misra, W. Gong, and D. Towsley Fluid-based Analysis of Network of AQM Routers Supporting TCP Flows with an Application to RED, Proc. ACM/SIGCOMM, 30 (2000), 151-160.

[21] H. Naito, T. Azuma, A. Nishimura, and M.Fujita, Experimental Verification of Congestion Controllers for TCP/AQM Networks, IEEJ Trans. EIS, 124 (2004), 2093-2100.

[22] Shevitz, D. and B. Paden, Lyapunov Stability Theory of Nonsmooth Systems, IEEE Trans. Autom. Control, 39 (9) (1994), 1910-1914.

[23] H. Wang, C. Liao, and Z. Tian, Effective adaptive virtual queue: a stabilising active queue management algorithm for improving responsiveness and robustness, IET Commun. 5 (2011), 99-109.

[24] P. Wang, H. Chen, X. Yang, and Y. Ma, Design and analysis of a model predictive controller for active queue management, ISA Trans. 51 (2012), 120-131.

[25] J. Xiong, and J. Lam, Stabilization of networked control systems with a logic ZOH, IEEE Trans. Autom. Control, 54 (2009), 358-363.

[26] H. Xu, A. Sahoo, and S. Jagannathan, Stochastic adaptive event-triggered control and network scheduling protocol co-design for distributed networked systems, IET Contr. Theory Appl. 8 (18) (2014), 2253-2265.

[27] Q. Xu, and J. Sun, A simple active queue management based on the prediction of the packet arrival rate, J. Network Comput. Appl. 42 (2014), 12-20.

[28] W. Zhang, M. S. Branicky, and S. M. Phillips, Stability of networked control systems, IEEE Control Syst. Mag. 21 (1) (2001), 84-99. 
[29] P. Zhang, C. Ye, X. Ma, Y. Chen, and X. Li, Using Lyapunov function to design optimal controller, J. Zhejiang Univ. Sci. A, 8 (2007), 113-118.

[30] L. Zhanga, and D. H. Varsakelis, Communication and control co-design for networked control systems, Automatica, 42 (2006), 953-958. 